\title{
An Auto-Modeling Method for Industrial Systems
}

\author{
Zhong Han ${ }^{1,2}$, Guicheng Zhang ${ }^{2}$, Lixing Yuan ${ }^{1}$ and Shengdun Zhao ${ }^{2}$ \\ ${ }^{1}$ School of Automation, Xi'an University of Posts \& Communications, Shaan'xi Province, China \\ ${ }^{2}$ School of Mechanical Engneering, Xi'an Jiaotong University, Shaan'xi Province, China \\ hzhong@stu.xjtu.edu.cn, sdzhao@mail.xjtu.edu.cn
}

\begin{abstract}
It is a difficult and valuable research to automatically generate the system model according to some rulers desired. So, a new auto-modeling method for industrial systems is presented. In the research, the industrial system is divided into many individual units, and some mathematical expressions are introduced for building the system model. A series of numbering rules for model nodes are defined in the light of individual unit coupling relationships. A data structure is designed to save the model information, and the saved data information is classified into many groups according to certain ruler and mapped to many classification sets. Next, these classification data in sets is continuously updated and done with by search operation and function calculation again and again. Further, each model elements are all assigned to relevant the layer and the column, and the industrial system model is gotten eventually. Finally, a real example is provided to verify the presented algorithm is feasible and can satisfy purpose requirements of availability, efficiency and accuracy. A layer-column type idea is shown in this algorithm realization, and this entire model is gradually generation. In addition, this algorithm also has a good universality, and can be widely applied into other large-scale manufacturing systems.

Index Terms - Modeling, Systems engineering, Data structure, Classification set
\end{abstract}

\section{Introduction}

The system model is often used as an effective tool to analyse and simulate reliability and safety problems in industrial systems. Models can provide a variety of means to help people to understand the change course of multi-media streaming motions in complex systems and the evolution process of some abnormal phenomena, such as the failure propagation track, multi-factor interactions, and sequence changes in time. At present, many system modeling tools have existed, some of them have its own specific application environment, but these peculiarities are limited to the wide application of these modeling methods. Reference [1] uses a graph-theoretic visualization approach to network risk analysis, A neural-network algorithm for a graph layout problem in [2], building three-dimensional path model in [3], specification of ontology for route graphs in [4], CNC machine tools reliability of intelligent network system control model and automatic generation of research in [5], Graph grammars generate the neural network method in [6]. It can be seen that figure modeling is widely used in various industries from these studies, that research of the model automatic generation algorithm is carried out by many industries, but an autogeneration algorithm for industrial system model is rare. So, a layer-column-type auto-generation algorithm for industrial system model is presented in this paper. The proposed algorithm can automatically generated a network model according to the system organizational structure in the inherent, and overcomes a lot of modeling conditions desired human participation to complete. In manual modeling, the workers are required must to have abundance industry know ledges. It is obviously inefficient used this modeling way. Eventually, the producing modeling will be inconsistent because the knowledge structure of the modeling personnel is different and learning the system is in many levels. In addition, this manual model can't reflect real-time changes of system date information, namely, the system models must also be followed dynamically changes with system structure changes.

Then, the layer-column-type auto-generation algorithm for the industrial system models will be narrated in detail from four aspects, modeling thought and expressions, the modeling algorithm, application examples, conclusions.

\section{Modeling Thought and Expressions}

\section{A. Modeling Analysis and Mathematical Expressions}

An industrial system can divided into more than one subunit through the decomposition, and there are many relationships between these sub-units. Therefore, it is very suitable that a mathematical graph $G$ is used to express the industrial system. A sub-unit of the system represents a node $v$ of the graph; a relationship between sub-units symbols an edge $e$ of the graph. The system units and the relationships between interrelated units are corresponded to the nodes and edges of the graph, respectively. This will eventually form an interconnected network. These nodes and edges is different each other by identifiers, the identifier of models represent and contains the attribute information they belong unit. Then, the mathematical expression of the system model is given as follows (1).

$$
G=\left(V, E \mid \quad v_{i} \ni V, \quad e_{j} \ni E \quad i=1 \cdots n, \quad j=1 \cdots m\right)
$$

Where, $v_{i}$ denotes the $i^{\text {th }}$ node of the model, $e_{j}$ denotes the $j^{\text {th }}$ edge.

Another expression of the industrial model is the relationship mark. A multidimensional vector sequence is used to save the relationship information of a model, such as expression (2). In terms of the industrial system, it is enough to reflect the situations of an entire industrial model, that some information must be preserved such as subsequent nodes, forward nodes, node relationships, and element identifier. The information vector sequence of relationship $\mathrm{R}$ is expressed as follows.

Model relationship $R$ (identifier $i d$, edge $e$, forward node 
$v_{s}$, subsequent node $v_{e}$, parameter $p_{k}$ ).

Mathematical expression is as follows.

$$
R_{s e}^{k}=\left(i d, e_{k}, v_{s}, v_{e}, p_{k}\right)
$$

Where, $R_{s e}^{k}$ denotes the $i^{\text {th }}$ relationship connected node $v_{s}$ and $v_{s}$

So that, $R$ is further simplified and represented with a sequence to facilitate later calculations and consider the purpose that $R$ are mainly reflected a sequence relation of between $v_{s}$ and $v_{e}$, the expression is as follows (3).

$$
R_{s e}^{k}=\left\langle v_{s}, v_{e}\right\rangle
$$

\section{B. Model Coding}

A large-scale system can produce a lot of individual units through the decomposition many times, and these units are corresponding to the nodes of the system model, respectively. To enhance the model usefulness, the node coding management must be adapted. It is found by investigating the operation of the existing system that most of the relationships between the system units are a one-way and irreversible, such as the current flow, water flow.

Therefore, the encoding of the nodes must meet the sequence requirements of the media coupling between the system nodes. The sequence maybe is the material flow orders, direction of current flows, system fault propagation paths, or system signal timing sequences.

In addition, according to the anomalies, the system partition degree is different in modeling processes. Some node granularity in [7] are parted into large, another node granularities are small. So, the model code must reflect these node relationships similar to the "cluster", and this node coding must be adjacent in same cluster.

The model coding must also ensure that the proposed method is solvable in dealing with engineering problems. Using the model for theoretical calculations need to ensure that the calculation result is convergent.

\section{Auto-Generation Model Processes}

\section{A. Layer-Column-Type Modeling Methods}

The layer-column-type algorithm idea can be used to automatically generate the industrial system model. Algorithms mainly include three aspect contents, this three aspect works are alternately carried out, and the industrial system model can be set up ultimately.

1) Processing Node Approach. The first node of models must be determined. In our research, the initial input devices of industrial systems are often regarded as the first nodes. Next, the initial node(s) is as the current node, the subsequent node of this current node is sought according to certain search algorithm, and the repeated node will be processed. Finally, when all nodes in whole node set are scanned and processed, up to now, the processing node part is end.

It is similar to a hierarchical process of nodes. The current nodes are divided and appointed a same layer in every processing turn, and the subsequent nodes are dispatched to the other one same layer. Here, the same level is also called the column. Nodes with same hierarchy are arranged in the node code sequence for the graphical representation, and can not be repeated. After each node is assigned and processed, the model structure of the entire industrial system will be basically fixed. So, this modeling approach based on the layer and column-based is named the layer-column-type modeling algorithm.

2) Processing Relationship Means. The relationship is a sequence in the mathematical expression, and is a line in the graphics form. Through analysing the model structure composition, two basic modern between any two nodes can be summarized. 1) The node connections of different layers see the (a) in Fig. 1, $i, j \ldots k$ are different node layer numbers, this connection between any two nodes is called the layer connection. 2) The node connections of the same level nodes see the (b) in Fig. 1. Then, this connection between any two nodes in the same layer is named the column connection. The entire industrial system model can be formed by any combinations of these two basic connection forms.

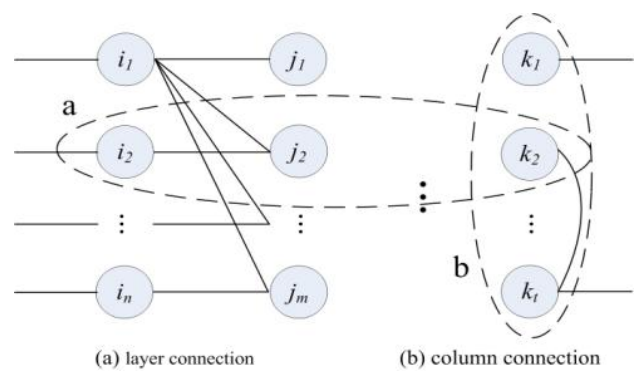

Fig. 1 Basic formations of node relationships

3) Processing Number Ways. Earlier the node number is introduced, and we know it is most important to make a series of number rules for nodes. Any node number in back layer must be greater than the forward tier that can be inferred in light of node number rules. These node numbers in the same level are appointed in term of number small on the above while number big on the following. The node and relationship labels are finished at same time, which will avoid the number label confusion caused by separately in producing the model.

\section{B. Modeling Calculation}

All computing operations are executed respectively on the basis of these data sets by this algorithm.

1) The set operation is often use in applications. When each search to node relationships between the sets will produce a Cartesian product operation, such as (4) and (5):

$$
\forall v_{x}, \forall v_{y} \in V \Rightarrow R(x, y)=\sum_{x, y} V_{x} \otimes V_{y} \subseteq V \otimes V=V^{2}
$$

More known:

$$
A \subseteq V^{2} ; B \subseteq V^{2} ; C \subseteq V^{2} ; D \subseteq V^{2}
$$

2) The data must be classified, and the results were stored in relevant vector space with the structure. Their calculation 
method is given as follow (6), (7), and (8):

$$
(A \neq \Phi \wedge B=\Phi) \rightarrow \exists\left(v_{x}, v_{y}\right) \in B \mid\left(B=\left\{A\left(v_{x}, v_{y}\right) \wedge v_{x}=v_{0}\right\}\right)
$$

Equation (6) effects an initial process of set $B$, these records of start node $v_{0}$ in set $A$ are assigned to set $B$, where, $v_{0}$ is with the smallest node number.

$$
C=\left\{\left(v_{x}, v_{y}\right) \mid \forall\left(v_{x}, v_{y}\right) \in A\left(v_{i}, v_{j}\right) \wedge B\left(v_{m}, v_{n}\right) \wedge v_{j}=v_{n}\right\}
$$

Equation (7) is an assignment process to set $C$. It is to save these records that are with the same subsequent nodes in set $A$ and set $B$ to the set $C$.

$$
D=\left\{C\left(v_{i}, v_{j}\right) \mid v_{i P}, v_{i t} \in V \wedge \forall v_{i p} \neq \forall v_{i t}\right\}
$$

By Equation (8), these records with the duplicate forward nodes in the set $C$ are deleted, and the remaining data in the set $C$ is assigned to the set $D$.

3) The algebra is used for the node location parameter calculation.

The calculation of horizontal position $x$, the calculating method is given by equation (9) as follows:

$$
x=x_{0}+\int_{1}^{t} a_{t} \quad 1 \leq t \leq l
$$

Where, $x_{0}$ is a position constant in the initial and horizontal direction; $a_{t}$ is the constants with an average distribution in horizontal direction, that is a jump step length of nodes in the horizontal direction; $t$ is the layer number of the current processing node in the horizontal direction; $l$ is tall layers in the horizontal direction.

The calculation of the vertical position y, calculating way is as follow (10):

$$
y=\int_{1}^{m} b_{m}+\left(y_{0}-\int_{1}^{n-1} b_{n-1}\right) / 2 \quad 1 \leq m \leq n
$$

Where, $b_{m}$ is an mean distribution constant in the vertical direction, is also a vertical step; $n$ is the total layer numbers that the presenting processing nodes is in the vertical direction. $y_{0}$ is the initial position in the vertical direction; $\mathrm{m}$ is the layer numbers of the current processing nodes in the vertical direction. Equation (10) ensures a symmetric distribution of nodes on the vertical direction along the center line.

\section{Modeling Procedure Processes}

The following is used the similar JAVA language to describe the algorithm implementation process, and gives some of the main program code of implementation methods.

1) In the initial, each vector structure space are empty, then, the classification methods are applied to load the data in each space, and the results are stored in the vector structure space A, B, C, D.

$\mathrm{A}=$ managerLocator.getMAManager()

.findByProperties (new String [] ["setAid"\}, new Object [] $\{$ setMid $\})$;

B.add (A.getAll $\left(v_{s}\right)$ ); // $s$ is a set of sequence nodes of the current processing nodes.
C.add (A.get (B.getAll $\left.\left.\left(v_{e}\right)\right)\right) ; / / e$ is a subsequent of the set B.

D.add (C. getAll $\left.\left(v_{u e}\right)\right) ; / / v_{u e}$ is an induplicate node.

2) The node position is worked out according to equation (9) and (10).

Assuming the current node position is $\left(x_{1}, y_{1}\right)$, and then generate the node position is as follows. sizes.

$\operatorname{G.fillOval}\left(x_{l}, y_{1}, \alpha, \beta\right) ; / / \alpha, \beta$ are parameters of node

3) Equation (9) and (10) are continuously used to calculate the location of the relationship. The relationships have the starting position calculation and the end position calculation. The calculation means is divided into two kinds of situation, edge position calculation for the same level and the edge position calculation with the different levels.

The edges with the same level have a same horizontal coordinate, $x_{2}=x_{1}$, and their vertical coordinate is computed by equation (10). This relationship often is expressed with the arc, and then the specific processing way is as follows:

G.draw $\operatorname{Arc}\left(x_{1}, y_{1}, x_{1}, y_{2}, E, F\right) ; / / E, F$ are the parameters to adjust arc sharps.

The computations of the start and end positions for the relationships at different levels, are obtained according to equation (9) and (10). Start-position coordinate is $\left(x_{1}, y_{1}\right)$, and end-position coordinate is $\left(x_{2}, y_{2}\right)$, then, this relationship is handled is as follows.

G.drawLine $\left(x_{1}, y_{1}, x_{2}, y_{2}\right)$;

\section{Cases}

This auto-generation algorithm for system models is applied in a real industrial system. This system is the energy and chemical system, these device combinations are complex, and this system has the higher safety requirements. The mapping table of model nodes and devices for this system sees TABLE I. The data structures of the system model relationship are stored in a defined TABLE II.

TABLE I Corresponding of Nodes and Equipments

\begin{tabular}{|c|c|l|}
\hline No. & Nodes & Equipments \\
\hline 1 & $v_{0}$ & Virtual start node \\
\hline 2 & $v_{1}$ & Turbine \\
\hline 3 & $v_{2}$ & Gearbox \\
\hline 4 & $v_{3}$ & Air filter \\
\hline 5 & $v_{4}$ & Compressor \\
\hline 6 & $v_{5}$ & Screwed valve 1 \\
\hline 7 & $v_{6}$ & Screwed valve 2 \\
\hline 8 & $v_{7}$ & Switch value 1 \\
\hline 9 & $v_{8}$ & Molecular sieve \\
\hline 10 & $v_{9}$ & Condenser 4 \\
\hline 11 & $v_{10}$ & Screwed valve 3 \\
\hline 12 & $v_{11}$ & Switch value 2 \\
\hline 13 & $v_{12}$ & Booster 1 \\
\hline 14 & $v_{13}$ & Condenser 3 \\
\hline 15 & $v_{14}$ & Booster 2 \\
\hline 16 & $v_{15}$ & Condenser 1 \\
\hline 17 & $v_{16}$ & Electromagnetic valve \\
\hline 18 & $v_{17}$ & Booster 3 \\
\hline 19 & $v_{18}$ & Condenser 2 \\
\hline
\end{tabular}


TABLE II Data Structure Formations

\begin{tabular}{|c|c|c|c|}
\hline Edge id & Edge name & Front node & Subsequent node \\
\hline 1 & $e_{0}$ & 1 & 2 \\
\hline 2 & $e_{1}$ & 1 & 4 \\
\hline 3 & $e_{2}$ & 2 & 3 \\
\hline 4 & $e_{3}$ & 3 & 5 \\
\hline 5 & $e_{4}$ & 4 & 5 \\
\hline 6 & $e_{5}$ & 5 & 6 \\
\hline 7 & $e_{6}$ & 5 & 7 \\
\hline 8 & $e_{7}$ & 5 & 8 \\
\hline 9 & $e_{8}$ & 6 & 9 \\
\hline 10 & $e_{9}$ & 8 & 10 \\
\hline 11 & $e_{10}$ & 9 & 11 \\
\hline 12 & $e_{11}$ & 10 & 12 \\
\hline 13 & $e_{12}$ & 11 & 13 \\
\hline 14 & $e_{13}$ & 12 & 13 \\
\hline 15 & $e_{14}$ & 13 & 15 \\
\hline 16 & $e_{15}$ & 13 & 14 \\
\hline 17 & $e_{16}$ & 14 & 15 \\
\hline 18 & $e_{17}$ & 15 & 16 \\
\hline 19 & $e_{18}$ & 15 & 18 \\
\hline 20 & $e_{19}$ & 16 & 18 \\
\hline 21 & $e_{20}$ & 16 & 17 \\
\hline 22 & $e_{21}$ & 18 & 19 \\
\hline 23 & $e_{22}$ & 17 & 19 \\
\hline
\end{tabular}

The system model shown in Fig. 2 is set up In accordance with the proposed algorithm to establish. This network model consists of 19 nodes, 23 edges, parameters, logo and other elements.

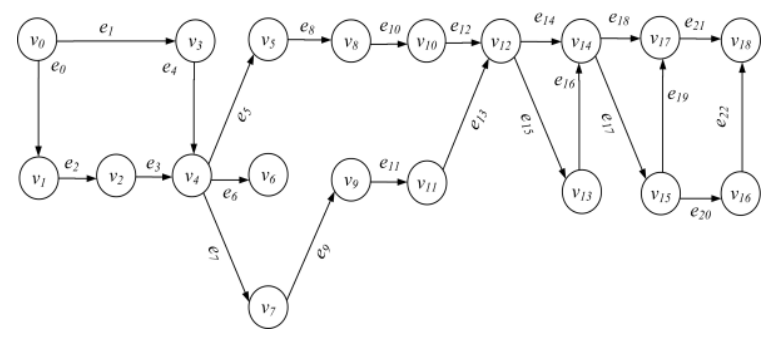

Fig. 2 A model made by auto-modeling methods

\section{Conclusions}

The presented algorithm greatly improves the modeling accuracies according to these means such as model node numbering, layer-column-type modeling, and multiple parameter calculation methods. It ensures the consistency and integrity of the model, avoids encountering errors by the artificial modeling in the previous, and improves the modeling efficiency. This algorithm can also caused the model a realtime and synchronization change when the system content is maintained and altered. It guarantees the synchronization of the system information and data, and this synchronization changes can prevent the occurred danger owing to "unknowable" system problems.

In addition, it is also very important to settle system problem using the suggesting methods combining some decision-making. Therefore, more effective application based on generated models for the system failure prediction, monitoring and maintenance will be the next step work.

\section{Acknowledgment}

This work is partially supported by the National Postdoctoral Science Foundation Granted numbered 2013M532033 and Industry-university-research Cooperation Project of Guangdong Province and Education Ministry Granted numbered 2011A090200060.

\section{References}

[1] O'HARE S, NOEL S, PROLE K, "A graph-theoretic visualization approach to network risk analysis," Visualization for Computer Security 5th International Workshop, pp. 60-67, 2008.

[2] ROBERT C, PAUL S, "A neural-network algorithm for a graph layout problem," IEEE Transactions on Neural Networks, vol. 7, pp. 341-345, 1996.

[3] HUANG Yaping, DU Jiangeng, CHEN Henry, "Construct threedimensional route from blueprint image of building," Chinese Journal of Beijin Jiaotong Universtity, 2010, 34(2): 79-83.

[4] Krieg-Bruckner B, Frese U, Luttich K, et al, "Specification of Ontology for Route Graphs,” Spatial Cognition IV LNCS3343, 2005:390-412.

[5] Xuejun Liu, Yazhou Jia, Riming Zhang, "Research on the control model and the automatically generation of cnc reliability intelligent network," Chinese Journal of Mechanical Engineering, vol. 39, pp. 114-117, 2003.

[6] CHAITANYA Vempati, MATTHEW Campbell, "A graph grammar approach to generate neural network topologies," 2007 Proceedings of the ASME International Design Engineering Technical Conferences and Computers and Information in Engineering Conference, v 6 PART A, 2008: 79-89.

[7] HAN Zhong, CHEN Fumin, GAO Zhiyong, GAO Jianmin, "Node granularity transformations based on object directed acyclic graph in system modeling," Journal of Xi'an Jiaotong University, 2008, 42(9): 1122-1126. 